\title{
Problems in Distortion Corrected Texture Classification and the Impact of Scale and Interpolation
}

\author{
Michael Gadermayr ${ }^{1}$, Michael Liedlgruber ${ }^{1},{\text { Andreas } \mathrm{Uhl}^{1} \text {, and Andreas Vécsei }}^{2}$ \\ 1 Department of Computer Sciences, University of Salzburg, Austria \\ \{mgadermayr, mliedl, uhl\}@eosy.sbg.ac.at \\ 2 St. Anna Children's Hospital, Endoscopy Unit, Vienna, Austria
}

\begin{abstract}
In the field of computer aided celiac disease diagnosis, wide-angle endoscopy lenses are employed which introduce significant barrel type distortions. Although the images can be rectified using distortion correction methods, computer based diagnosis suffers from missing information in highly distorted image regions. First, we investigate the impact of simple and advanced interpolation techniques on the classification rates. Furthermore we explore the effect of considering different image resolutions. Whereas in previous studies distortion correction in most cases turned out to be disadvantageous, we show that for certain setups distortion correction definitely is advantageous.
\end{abstract}

Keywords: Distortion correction, endoscopy, classification, celiac disease.

\section{Introduction}

Celiac disease [11] is an autoimmune disorder which affects the small intestine in genetically predisposed individuals after introduction of gluten containing nutrient. Characteristic for this disease is an inflammatory reaction in the mucosa of the small bowel caused by a dysregulated immune response triggered by ingested gluten proteins. During the course of celiac disease the mucosa loses its absorptive villi and hyperplasia of the enteric crypts occurs leading to a diminished ability to absorb food. According to a study [2], the overall prevalence in the USA in not-at-risk groups was 1:133 .

Computer aided celiac disease diagnosis relies on images taken during endoscopy. The employed cameras are equipped with wide angle lenses, which suffer from a significant amount of barrel type distortion. Whereas the distortion in central image pixels can be neglected, peripheral regions are highly distorted. Thereby, the feature extraction as well as the following classification is compromised. Based on camera calibration, distortion correction (DC) techniques are able to rectify the images. However, although the barrel type distortion can be undone, especially in peripheral regions there remains a lack of information, as the DC method stretches the image. The lack of information has to be compensated using an interpolation technique.

In recent studies, the impact of barrel type distortion [9] and distortion correction [5] on the classification rate of celiac disease endoscopy images was investigated. The authors have shown that image patches in peripheral regions, which are more strongly affected by the distortion are more likely to be misclassified. However, with distortion 
correction, the classification rate on average even suffers. In [4], different distortion correction techniques have been investigated.

In this paper, priority is given to the following aspects:

- Interpolation: First, different interpolation methods are investigated. Not the visual quality, but only the classification rate is our interest. Apart from the simple and commonly used bilinear and nearest neighbor interpolation, we investigate a Lanczos filter which effectively imitates the perfect low pass filter. Moreover, a (non-linear) edge preserving method is analyzed.

- Scale: Furthermore, we investigate the impact of the image scale on the classification rate. For all features a setup is chosen, that only pixels in the 8-pixel neighborhood are considered. These features are adjusted implicitly, by downscaling the input image. We know that distortion correction in a small neighborhoods (achieved with the original image), mostly does not improve the performance. However, we suspect that by downscaling the image, the profit of distortion correction could be increased and thereby a benefit in the classification performance might be achieved.

The paper is organized as follows: In Sect. 2, the problems of DC based feature extraction are explained. In Sect. 3, the utilized DC method, the different interpolation methods and the used features are explained. In Sect.4 experiments are shown and the results are discussed. Section 5 concludes this paper.

\section{Motivation - Problems within Distortion Correction}

Distortion correction of barrel type distortions is known to effectively rectify the geometrical properties of images taken with wide angle or fish eye lenses. Visually, the distortion corrected images seem to be better suited for classification of computer based celiac disease diagnosis than the distorted images. However, there are certain inadequacies which arise if DC is applied. Whereas the images are geometrically corrected, in case of considering small details (i.e. texture), new problems are introduced.

Intuitively, the probability of a feature or of single characteristics of a feature should be uniformly distributed over all coordinates of the image. For example this condition could be violated in case of systematic sensor faults. Distortion correction, is another source for a violation of the mentioned condition.

We utilize images taken with the same endoscope and we compute pixel based properties (which are also included in features), for each pixel in all images. Afterwords, the average of the features for each coordinate over all images is calculated, to get the mean for each point. Having a large number of images, the resulting data should be approximately homogeneous. A high degree on inhomogeneity indicates an anomaly.

As an example, we visualize a property which is a part of the well known local ternary patterns (LTP [14]). Especially, we consider the probability that the pixel above the current pixel has approximately $( \pm 2)$ the same value as the current pixel. In Fig.1, the distributions of this certain property are given for the original (Fig. 1a) and for the distortion corrected image (with different downscaling factors: Fig. 1b 1d). A similar behavior can be achieved not just with the mentioned property, but with many others too (e.g. LBP [12], ELBP [8]). In the original image, apart from slight stripes (caused by 


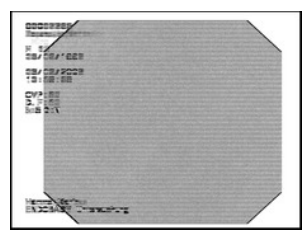

(a) Original image

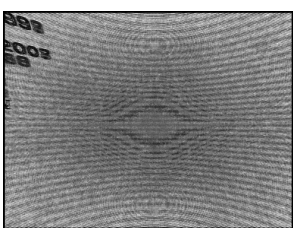

(b) DC image (original size)

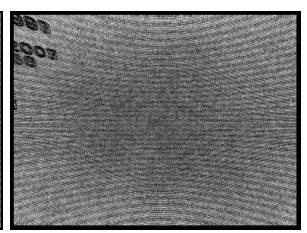

(c) DC image (downscaling factor 2)

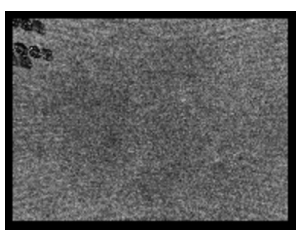

(d) DC image (downscaling factor 4)

Fig. 1. Distribution of the upper sample of local ternary patterns: Especially we consider the occurrences of the sample value equaling approximately $( \pm 2)$ the center value

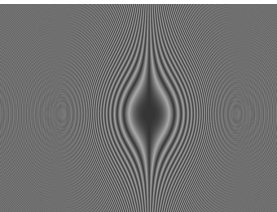

(a) $\mathrm{x}$-Distance

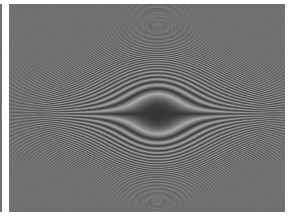

(b) y-Distance

Fig. 2. The distances to the next sample point in interpolation within DC are represented by gray values. A dark region corresponds to a small distance and vice versa.

the sensor), the property is approximately uniformly distributed. In the DC images, the property definitely is not approximately uniformly distributed, however, with downscaling the uniform distribution can be recovered. With blurring after applying the distortion correction, a similar behavior can be achieved. For these experiments, we utilized bilinear interpolation within distortion correction as done in previous studies [9]5|4]. We identified two major issues which occur in distortion correction:

\section{- Interference patterns}

Especially in central, but also in peripheral regions strange (interference) patterns can be observed (Fig. 1b).

Explanation: During interpolation (needed in rasterization), some required pixel values are near to real sample pixel values in the original image. In opposite, others are approximately in the middle of four sample pixels. The problem in distortion correction is the following: Whereas in the first case, edge frequencies are maintained quite effectively, in the second case the edges are blurred during interpolation. In Fig. 2 the distances of interpolation coordinates to the next sample coordinates are visualized. It can be seen, that the pattern shown in Fig. $2 b$ can also be recognized in the averaged feature images (Fig. 1b.

\section{- Contrast between center and peripheral regions}

Apart from the mentioned patterns, a significant difference between central and peripheral regions can be observed with some feature properties.

Explanation: In central image regions, the image is not changed significantly within

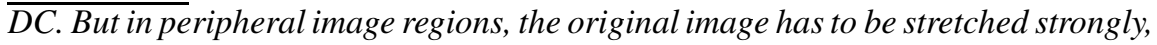
in order to rectify the barrel type distortion. The stretching results in a decrease of 
high frequencies which is visualized in Fig. 3. These figures show the average local frequencies, contained in the distorted (Fig. 3a) and in the distortion corrected images (Fig. 3b) with reference to the distance to the center of distortion. Therefore, we computed the local discrete Fourier transform (with window size $16 \times 16$ ) for all of our endoscopy images and for all pixels. Then we averaged all Fourier spectra having the same distance to the center of distortion. In Fig. $3(a)$ and $(b)$ for each frequency (corresponds to a ring in the 2-D Fourier domain) on the $x$-axis and for each distance to the center of distortion (y-axis), the logarithmic average value of the Fourier power spectrum is given. In order to emphasize on the differences, in Fig. $3 c$ we subtracted the distortion corrected from the distorted frequency image. In this image, it can be seen, that with an increasing distance to the center of distortion, especially high frequencies are decreasing in case of distortion correction.

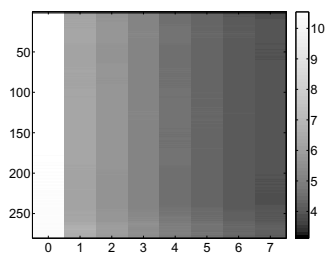

(a) Distorted Image

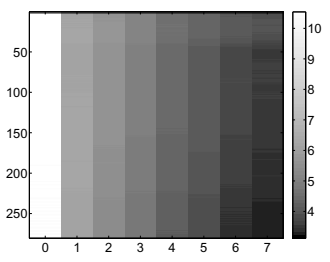

(b) DC Image

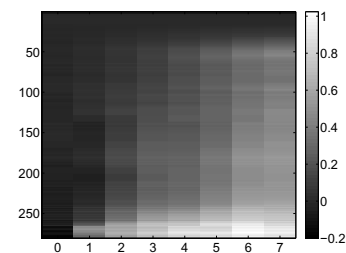

(c) Difference

Fig. 3. Local DFT: These figures show averaged local frequencies. On the $x$-axis the frequencies (a low value corresponds with a low frequency and vice versa) and on the y-axis the distances from the center of distortion are given.

\section{Theory}

\subsection{Distortion Correction}

We utilize the distortion correction method based on the work of Melo et al. [10]. In this approach, the circular barrel type distortion is modeled by the division model [3]. Having the center of distortion $\hat{x}_{c}$ and the distortion parameter $\xi$, an undistorted point $x_{u}$ can be calculated from the distorted point $x_{d}$ as follows:

$$
x_{u}=\hat{x}_{c}+\frac{\left(x_{d}-\hat{x}_{c}\right)}{\left\|x_{d}-\hat{x}_{c}\right\|_{2}} \cdot r_{u}\left(\left\|x_{d}-\hat{x}_{c}\right\|_{2}\right) .
$$

$\left\|x_{d}-\hat{x}_{c}\right\|_{2}$ (in the following $r_{d}$ ) is the distance (radius) of the distorted point $x_{d}$ from the center of distortion $\hat{x}_{c}$. The function $r_{u}$ defines for a radius $r_{d}$ in the distorted image, the new radius in the undistorted image:

$$
r_{u}\left(r_{d}\right)=\frac{r_{d}}{1+\xi \cdot r_{d}^{2}} .
$$

Figure 4 shows a distorted and the corresponding undistorted image of a checkerboard pattern. 


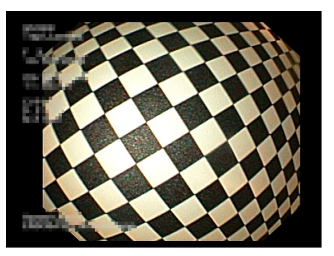

(a) Original image

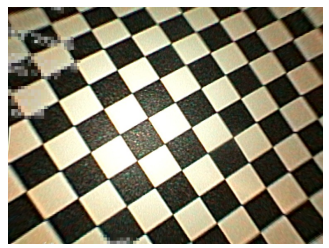

(b) DC image

Fig. 4. Distorted and undistorted image of a (planar) checkerboard pattern

\subsection{Interpolation Techniques in Distortion Correction}

In order to get a rasterization after applying a distortion correction method, an interpolation method must be applied. We investigate the following techniques, which are identified to have quite different properties:

\section{- Bilinear interpolation}

\section{- Nearest neighbor interpolation}

\section{- Lanczos interpolation}

The Lanczos filter [1] with $a=3$ is known to imitate the perfect low pass filter (sinc) quite effectively and reduces ringing artifacts. With this filter, the available frequencies are retained, whereas aliasing is avoided.

\section{- Edge preserving interpolation}

The Lanczos filter is known to retain the available frequencies. However, in this case due to the stretching of the image in peripheral regions, high frequencies (sharp edges) are missing after undistortion. Consequently, maintaining the available frequencies is not enough to reconstruct the real edge information. Therefore, an edge preserving interpolation method [15] has been implemented. Unlike usual (linear) interpolation kernels, with this nonlinear approach the behavior of the interpolation depends on the image properties. Near edges, high frequencies are encouraged (similar to nearest neighbor interpolation), whereas smooth regions are retained smooth (similar to linear interpolation).

In Fig. 5] a perfect (undistorted) checkerboard pattern (Fig. 5a-5d) and a smooth gray value gradient image (Fig. 5e-5h) are undistorted (leads to an inverse distortion) and the different interpolation techniques are applied. Whereas from the first images, the ability of edge preservation, from the second image the ability of preserving smooth gradients can be deduced visually. Although undistorting an undistorted image is not a sensible application, the differences of the interpolation methods can be seen well with artificial images. The major positive $(+)$ and negative $(-)$ properties of the interpolation techniques are outlined in Table 1. It can be seen, that the chosen methods have quite different strengths and weaknesses. 


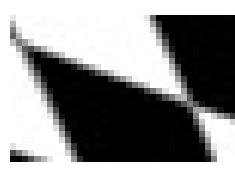

(a) Bilinear

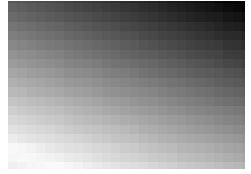

(e) Bilinear

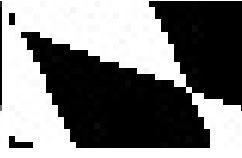

(b) Nearest $n$.

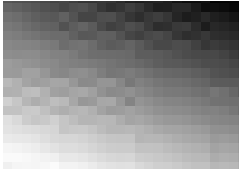

(f) Nearest $n$.

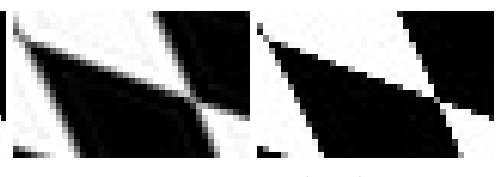

(c) Lanczos

(d) Edge pres.

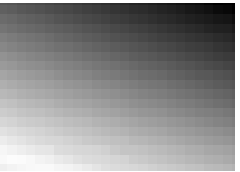

(g) Lanczos

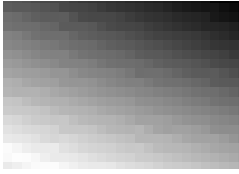

(h) Edge pres.

Fig. 5. The impact of the different interpolation methods on a distorted checkerboard pattern (top row) and a smooth gray value gradient image (bottom row)

Table 1. Properties of interpolation methods

\begin{tabular}{|l|c|c|c|c|}
\hline & Bilinear & Nearest n. & Lanczos & Edge pres. \\
\hline edge preservation & - & + & 0 & + \\
smoothness & + & - & + & 0 \\
avoid ringing & + & + & 0 & + \\
\hline
\end{tabular}

\subsection{Features in Classification}

In order to investigate the feature extraction methods with differently downscaled images, all of our features are adjusted, to operate only in the direct neighborhood (only the 8 neighbors are considered). For the experiments, the following features are used:

- Local binary patterns [12] (LBP): This features is used with a radius (i.e. the distance to the neighboring samples) of 1 and $4\left(\mathrm{LBP}_{4}\right)$ and $8\left(\mathrm{LBP}_{8}\right)$ neighboring samples, respectively.

- Local ternary patterns [14] (LTP): This feature is used with a radius of 1 and with 8 neighbors. The threshold, which has been evaluated during exhaustive search, is set to 1 .

- Extended local binary patterns [8] (ELBP): As LBP, ELBP is used with a radius of 1 and 8 neighbors.

- Gray level co-occurrence matrix [6] (GLCMP): The feature vector consists of the Haralick features [6] contrast, correlation, energy and homogeneity of the gray level co-occurrence matrices with different offset vectors. In order to focus on small neighborhoods (as above), the offset vectors $(0,1)^{T},(1,0)^{T},(1,1)^{T},(1,-1)^{T}$ have been chosen for creating the matrices.

- Edge co-occurrence matrix [13] (ECM): To get the ECM, first the orientations of all local image gradient quantized to 8 directions have to be evaluated. Whereas non-edge pixels (this is determined using the canny-edge detector) are ignored, with the orientation-labels ( 1 to 8 ) of edge pixels, the gray level co-occurrence matrix is computed (as above). As ECM feature vector the whole matrix is used. 


\section{Experiments}

\subsection{Experimental Setup}

The image test set used contains images of the duodenal bulb taken during duodenoscopies at the St. Anna Children's Hospital using pediatric gastroscopes (with resolution $768 \times 576$ and $528 \times 522$ pixels, respectively). In a preprocessing step, texture patches with a fixed size of $128 \times 128$ pixels were manually extracted. The size turned out to be optimally suited in earlier experiments on automated celiac disease diagnosis [7]. In case of distortion correction, the patch position is adjusted according to the distortion function. Downscaling with bicubic interpolation is executed after patch extraction (i.e. the considered regions are always the same, only the resolution differs). To generate the ground truth for the texture patches used, the condition of the mucosal areas covered by the images was determined by histological examination from the corresponding regions. Severity of villous atrophy was classified according to the modified Marsh classification scheme [11]. Although it is possible to distinguish between the different stages of the disease (called Marsh 3A-3C), we only aim in distinguishing between images of patients with (Marsh3A-3C) and without the disease (called Marsh0). We decided for this policy, because the two classes case is more relevant in practice. Our experiments are based on a database containing 163 (Marsh 0) and 124 (Marsh 3A-3C) images, respectively. Example texture patches are shown in Fig. 6. For classification, we use the k-nearest-neighbor classifier. This rather weak classifier has been chosen to emphasize on quantifying the discriminative power of the features proposed in this work. To avoid any bias in the results, leave-one-patient-out cross validation is utilized. We evaluated all different combinations of downscaling factors and interpolation techniques. We also considered previously blurring in combination with downscaling, but thereby the achieve classification results can not be improved. Moreover, different interpolation techniques within image resizing did not lead to significantly different rates.

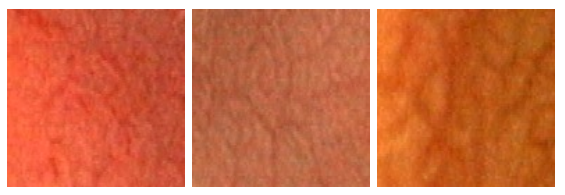

(a) Marsh 3A-3C

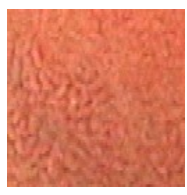

(b) Marsh 0

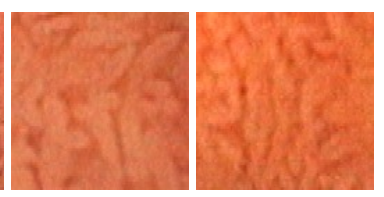

Fig. 6. Example patches of patients with (left) and without the disease (right)

\subsection{Results}

In Fig. $7 \mathrm{a}-7 \mathrm{f}$, the achieved classification rates with the different features and different image resolutions can be observed. Whereas the bold lines represent the approach without distortion correction, the different thin lines stand for different interpolations within distortion correction. In all figures can be seen, that the original approach (i.e. without distortion correction) is beneficial in case of the original image size (left most points). However, with increasing downscaling factor, in general the classification rates 


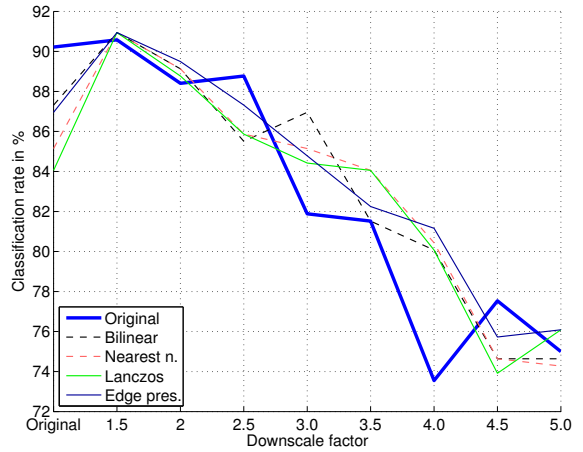

(a) $\mathrm{LBP}_{8}$ (8 sample points)

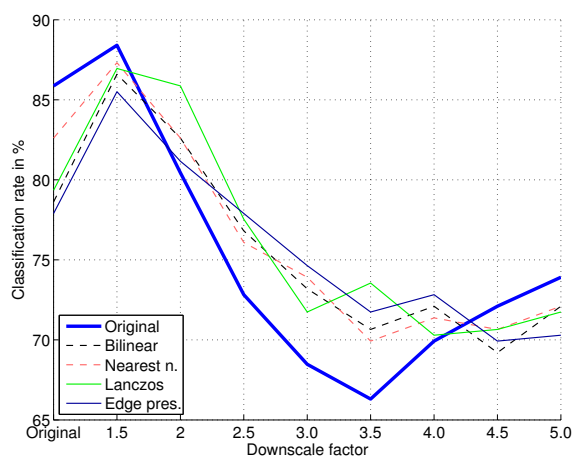

(c) ELBP

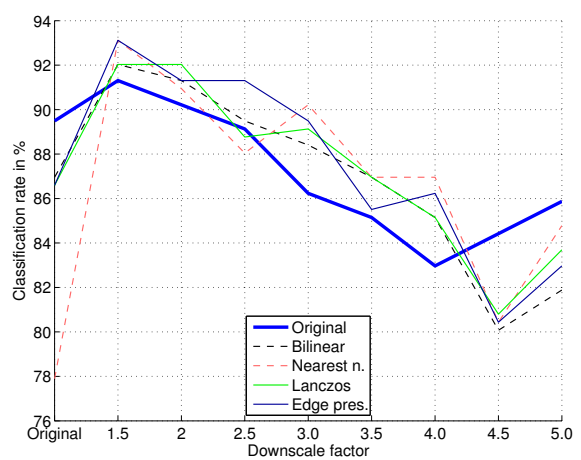

(e) LTP

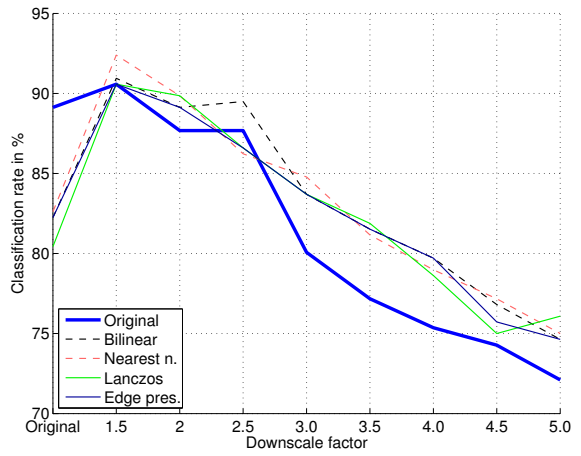

(b) $\mathrm{LBP}_{4}$ (4 sample points)

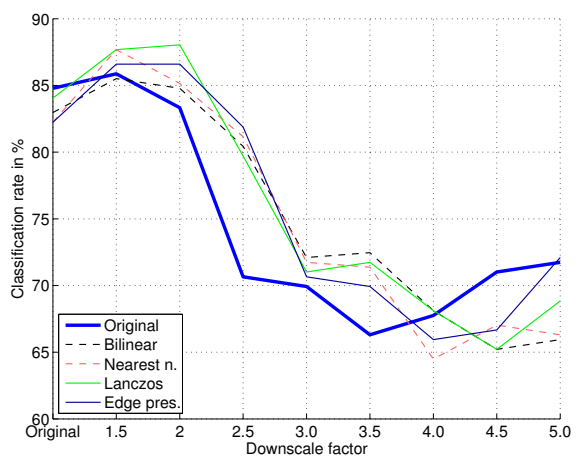

(d) ECM

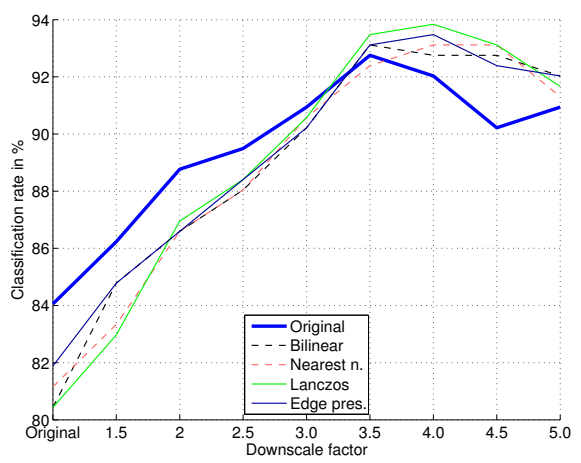

(f) GLCMP

Fig. 7. The plots show classification rates achieved with the considered features. Especially, the dependency of the scaling factor (x-axis) and the different interpolation methods (thin lines) can be seen.

with distortion correction are increasing compared with the approach without DC. For each feature, downscaling factors exist, for which DC is beneficial (points where all thin lines are above the thick line). If the discriminative power of the feature benefits 
from the slight image downscaling (factors 2 and above), then the distortion correction has a positive effect on the classification rate as shown in Fig. 7b, 7d, 7e and 7f, With the features $\mathrm{LBP}_{4}$, ECM and LTP the best overall classification rates are achieved with distortion correction and a moderate downscaling factor (between 1.5 and 2.0). With GLCMP, the best rate is achieved with distortion correction and a high downscaling factor (4.0). Especially using the GLCMP feature, a considerable performance increase (considering all downscaling factors) can be observed. Otherwise, if the classification rate falls significantly with the downscaling factor, the best classification rate is achieved without DC (Fig. 7c With ELBP the classification rate strongly falls between downscaling factors 1.5 to 3 ).

\subsection{Discussion}

A positive effect of distortion correction can be observed, especially when the images are downscaled. Downscaling the image implies, that pixels which a higher displacement (in the original image) are considered. Consequently, high frequencies (i.e. sharp edges) in the image are no longer extracted. Instead, with the downscaled images, lower frequencies are extracted by the feature. With the original images, distortion correction in general does not provide better results. A reason for this behavior is, that the regarded high frequencies are destroyed by the image stretching of the DC method in peripheral regions. With downscaled images, the geometric context and low frequencies are getting more important than high frequencies (sharp edges). Therefore, the distortion correction has a beneficial effect on the classification rate. The enhanced interpolation methods do not imply significantly better classification results. As distortion correction on original images is disadvantageous, the missing information cannot be compensated effectively even by the enhanced methods.

\section{Conclusion}

If images are downscaled, a positive effect of distortion correction can be observed. That means, distortion correction is especially sensible, if the classification rate utilizing a specific feature benefits from the downscaling (e.g. with the gray level co-occurrence matrix). Using feature extraction methods which suffer strongly from downscaling (e.g. ELBP), distortion correction does not improve the best classification result. The difference between the interpolation techniques is quite small. Even with the more sophisticated methods (the Lanczos and the edge preserving method [15]) no significant improvement of classification rates can be achieved (compared to the very simple bilinear and nearest neighbor interpolation). We showed that high frequencies cannot be maintained with any interpolation method, whereas the lower frequencies are maintained with each of the investigated interpolation techniques.

Acknowledgments. This work is partially funded by the Austrian Science Fund (FWF) under Project No. 24366. 


\section{References}

1. Duchon, C.E.: Lanczos filtering in one and two dimensions. J. Appl. Meteor. 18(8), 1016$1022(1979)$

2. Fasano, A., et al.: Prevalence of celiac disease in at-risk and not-at-risk groups in the united states: A large multicenter study. Archives of Internal Medicine 163(3), 286-292 (2003)

3. Fitzgibbon, A.W.: Simultaneous linear estimation of multiple view geometry and lens distortion. In: CVPR, pp. 125-132 (2001)

4. Gschwandtner, M., Hämmerle-Uhl, J., Höller, Y., Liedlgruber, M., Uhl, A., Vécsei, A.: Improved endoscope distortion correction does not necessarily enhance mucosa-classification based medical decision support systems. In: Proc. of the Intern. Workshop on Multimedia Signal Processing (MMSP 2012), pp. 158-163 (September 2012)

5. Gschwandtner, M., Liedlgruber, M., Uhl, A., Vécsei, A.: Experimental study on the impact of endoscope distortion correction on computer-assisted celiac disease diagnosis. In: Proc. of the 10th Intern. Conf. on Information Technology and Applications in Biomedicine (ITAB 2010) (November 2010)

6. Haralick, R.M., Shanmugam, K., Dinstein, I.: Textural features for image classification. IEEE Trans. on Systems, Man, and Cybernetics 3, 610-621 (1973)

7. Hegenbart, S., Kwitt, R., Liedlgruber, M., Uhl, A., Vécsei, A.: Impact of duodenal image capturing techniques and duodenal regions on the performance of automated diagnosis of celiac disease. In: Proc. of the 6th Intern. Symp. on Image and Signal Proc. and Analysis (ISPA 2009), pp. 718-723 (September 2009)

8. Liao, S., Zhu, X., Lei, Z., Zhang, L., Li, S.Z.: Learning multi-scale block local binary patterns for face recognition. In: Lee, S.-W., Li, S.Z. (eds.) ICB 2007. LNCS, vol. 4642, pp. 828-837. Springer, Heidelberg (2007)

9. Liedlgruber, M., Uhl, A., Vécsei, A.: Statistical analysis of the impact of distortion (correction) on an automated classification of celiac disease. In: Proc. of the 17th Intern. Conf. on Digital Signal Processing (DSP 2011), Corfu, Greece (July 2011)

10. Melo, R., Barreto, J.P., Falcao, G.: A new solution for camera calibration and real-time image distortion correction in medical endoscopy-initial technical evaluation. IEEE Trans. Biomed. Eng. 59(3), 634-644 (2012)

11. Oberhuber, G., Granditsch, G., Vogelsang, H.: The histopathology of celiac disease: time for a standardized report scheme for pathologists. European Journal of Gastroenterology and Hepatology 11, 1185-1194 (1999)

12. Ojala, T., Pietikäinen, M., Harwood, D.: A comparative study of texture measures with classification based on feature distributions. Pattern Recognition 29(1), 51-59 (1996)

13. Rautkorpi, R., Iivarinen, J.: A novel shape feature for image classification and retrieval. In: Proc. of the International Conference on Image Analysis and Recognition (ICIAR), pp. 753760 (2004)

14. Tan, X., Triggs, B.: Enhanced local texture feature sets for face recognition under difficult lighting conditions. In: Zhou, S.K., Zhao, W., Tang, X., Gong, S. (eds.) AMFG 2007. LNCS, vol. 4778, pp. 168-182. Springer, Heidelberg (2007)

15. Wang, F., Xu, Y., Zhao, Y., Hu, F.: A new nonlinear interpolation algorithm for edge preserving. In: 2010 International Conference on Multimedia Technology (ICMT), pp. 1-4 (October 2010) 